\title{
Lower Aorto-Iliac Bifurcation Position and Incident Cardiovascular Disease: A Multi-Ethnic Study of Atherosclerosis (MESA)
}

\author{
Nketi I. Forbang, MD, MPH ${ }^{1 *}$, Matthew A. Allison, MD, $\mathrm{MPH}^{1,2}$, Michael H. Criqui, MD, MPH ${ }^{1}$ \\ 1 Department of Family Medicine and Public Health, University of California, San Diego, La Jolla, California, USA \\ 2 Department of Vascular Surgery, San Diego Veterans Administration Health Care Systems, La Jolla, California, USA
}

\begin{abstract}
Background: With increasing age, a downward shift of the aorto-iliac bifurcation relative to the lumbar spine occurs. A lower bifurcation position is an independent marker for adverse vascular aging and is associated with increased burden of cardiovascular disease (CVD) risk factors; however, the associations between lower bifurcation position and CVD events remain unknown.

Methods: Abdominal computed tomography scans were used to measure the aorto-iliac bifurcation distance (AIBD, distance from the aorto-iliac bifurcation to the L5/S1 disc space). Cox proportional hazard analysis was used to determine the independent hazard of a lower bifurcation position (smaller AIBD) for incident coronary heart disease (CHD, defined as myocardial infarction, resuscitated cardiac arrest, or sudden cardiac death), CVD (CHD plus stroke or stroke death), and allcause mortality (ACM).

Results: In the 1,711 study participants ( $51 \%$ male), the mean AIBD was $26 \pm 15 \mathrm{~mm}$. After a median follow-up of 10 years, $63(3.7 \%)$ developed CHD, 100 (5.8\%) developed CVD, and 129 (7.5\%) were deceased. Compared to the $4^{\text {th }}$ quartile of AIBD (highest bifurcation position), participants in the $1^{\text {st }}$ quartile (lowest bifurcation position) had increased risk for CHD (hazard ratio $(H R)=$ 1.5, 95\% confidence interval (CI): 0.8-3.0, $P=0.2)$, CVD (HR = 1.8, 95\% Cl: 0.9-2.7, P = 0.1), and ACM (HR = 2.2, 95\% Cl: 1.3-3.6, $P=0.01$ ). After adjustments for CVD risk factors, the HR for ACM was no longer significant.
\end{abstract}

Conclusion: Despite being an independent marker for adverse vascular changes in the aorta, a lower aorto-iliac bifurcation position was not independently associated with future CVD events. The opposing effects of atherosclerosis and stiffness in the aorta may, in part, explain our null findings.

Copyright $\odot 2016$ Science International Corp.

\section{Key words}

Aorto-iliac bifurcation distance - Cardiovascular disease events • Atherosclerosis arterial stiffness

\section{Introduction}

The aorto-iliac bifurcation distance (AIBD) is the distance $(\mathrm{mm})$ from the aorto-iliac bifurcation to the L5/S1 disc space and is used to determine the position of the bifurcation relative to the lumbar spine. Thus, a larger AIBD indicates a higher bifurcation position, while a smaller AIBD indicates a lower bifurcation position. In two separate cohorts ( $n=748, n=1,711$ ), we found a lower bifurcation position with increasing age, independent of cardiovascular disease (CVD) risk factors [1, 2]. In the Multi-Ethnic Study of Atherosclerosis (MESA), we additionally found that atherosclerotic risk factors of male gender, smoking, and hypertension were associated with a lower bifurcation position [2]. (c) 2016 AORTA

Published by Science International Corp. ISSN 2325-4637

Fax +1 2037853552

E-Mail: aorta@scienceinternational.org

http://aorta.scienceinternational.org
Accessible online at:

http://aorta.scienceinternational.org
* Corresponding Author:

Nketi I. Forbang, MD, MPH

Department of Family Medicine and Public Health

University of California, San Diego

9500 Gilman Drive, MC 0965 La Jolla, California, 92093, USA

Tel.: +1 858822 7680, Fax: +1 858822 7662, E-Mail: nforbang@gmail.com 
In contrast, diabetes and elevated triglycerides, which are commonly associated with arterial stiffness, were associated with a higher bifurcation position. Associations of a lower bifurcation position with incident CVD events, however, remain unknown. Thus, we sought to determine the associations between a lower bifurcation position and future coronary heart disease (CHD), which was defined as myocardial infarction, resuscitated cardiac arrest, sudden cardiac death; CVD, which was defined as CHD plus stroke and stroke death; and all-cause mortality (ACM).

\section{Materials and Methods}

\section{Study Design and Participants}

A detailed description of the MESA study design has been published previously [3]. In brief, MESA is a multicenter, prospective cohort designed to investigate the epidemiology of atherosclerosis. Between July 2000 and August 2002, 6814 men and women (age 45-84 y), who were of Caucasian, Hispanic, African, and Chinese ethnicity and were free from clinically manifest CVD, were recruited for baseline visits at six US field centers: New York, New York; Baltimore, Maryland; Winston-Salem, North Carolina, St. Paul, Minnesota; Chicago, Illinois; and Los Angeles, California.

During follow-up visits between August 2002 and September 2005, 2,202 MESA participants who were representative of the study population were asked to participate in an ancillary study that focused on abdominal aortic calcium. Of these, 2,172 agreed to participate. Individuals were excluded if they were premenopausal or had a recent abdominal computed tomography (CT) scan. The AIBD was measured in 1,711 participants with identifiable bifurcations and L5-S1 disk spaces on CT (method below). Signed informed consent was obtained from all participants, and institutional review board approval was obtained from all participating institutions.

\section{CVD Events}

A detailed description of the adjudication process for CVD events has been previously published [3]. Briefly, participants or their next of kin (if participants were unavailable) were contacted at intervals of 9-12 months by telephone, and trained interviewers inquired about interim hospital admissions, cardiovascular outpatient diagnoses, and death. Medical records and death certificates were requested for verification. Two physicians blinded to participants' risk factors reviewed the data, classified CVD events, and assigned incidence dates. If disagreements persisted after adjudication, a full mortality and morbidity review committee made the final classification. For the current study, CVD events included CHD (myocardial infarction, resuscitated cardiac arrest, or CHD death, CVD (CHD plus non-fatal or fatal stroke), and ACM.

\section{AIBD}

Procedures for AIBD measurement have previously been published [2]. Briefly, computer software (Osiris 4.19; University of Geneva, Geneva, Switzerland) was used to identify the coordinates in the $x-, y-$, and $z$-planes for the aortic bifurcation and the L5-S1 disk space. A straight distance between the aortic bifurcation and the L5-S1 disc space was measured as the difference of the $z$-plane coordinates (interclass correlation = 0.89 ; Figure 1).

\section{Risk Factor Assessment}

Participants completed standardized questionnaires at baseline to obtain information on demographics, medical history, and smoking history. A medication inventory was performed, and patients were classified by their use of antihypertensive or hypoglycemic medications. Systolic blood pressure (SBP) was measured three times in the seated position with a Dinamap model Pro 100 automated oscillometric sphygmomanometer (Critikon, Tampa, FL, USA). The mean of the final two measurements was used. Blood samples were obtained after a 12-h fast for the measurements of total cholesterol and high-density lipoprotein (HDL) cholesterol. Diabetes was defined as fasting plasma glucose $>126 \mathrm{mg} / \mathrm{dL}$ or use of hypoglycemic medications.

\section{Statistical Analysis}

Cohort characteristics were summarized by means (SD) and proportions. Analysis of variance was used to compare patient characteristics. Kaplan-Meier curves and log-rank tests were used to investigate differential survival among AIBD quartiles. Cox proportional hazard regression was used to examine the association of AIBD (continuous and quartiles) for incident CHD, CVD, and ACM. Model 1 was unadjusted, and model 2 was adjusted for age, gender, ethnicity, smoking, diabetes, SBP, hypertension medication use, and total and HDL cholesterol. All analyses were conducted using PASW Statistics 20 (IBM Corp. 2011 Armonk, IL, USA). P-values < 0.05 (two-sided) were considered significant.

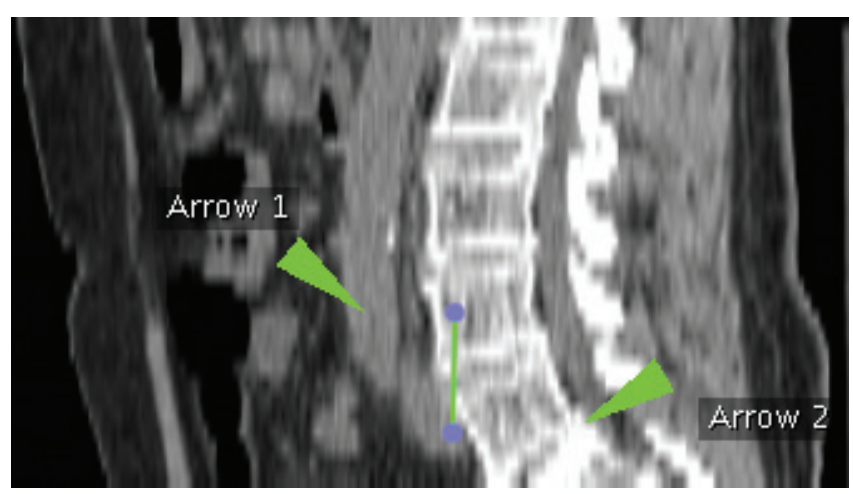

Figure 1. Sagittal computed tomography image of the abdomen. Arrow 1 = aorto-iliac bifurcation. Arrow 2 = posterior L5-S1 disc space. Vertical line $=$ aorto-iliac bifurcation distance (AIBD), which was calculated from coordinates (X,Y, and Z) of arrows 1 and 2 . 


\section{Results}

In the 1,711 participants ( $51 \%$ male), the mean age was $65 \pm 10 \mathrm{y}$, and the mean AIBD was $26 \pm$ $15 \mathrm{~mm}$. Compared to the $4^{\text {th }}$ AIBD quartile (highest bifurcation position), the $1^{\text {st }}$ AIBD quartile (lowest bifurcation position) had more smokers and higher SBP (Table 1). In our cohort, 63 (3.7\%) CHD events, 100 (5.8\%) CVD events, and 129 (7.5\%) total deaths occurred (Table 2). The log-rank survival tests among AIBD quartiles for $\mathrm{CHD}\left(\chi^{2}=2.1, P=0.05\right)$, $\operatorname{CVD}\left(\chi^{2}=3.8, P=0.3\right)$, and $\operatorname{ACM}\left(\chi^{2}=15, P<0.01\right)$ are shown in Table 2. The Kaplan-Meier curves of AIBD quartiles for ACM are shown in Figure 1. The $1^{\text {st }}$ AIBD quartile (lowest bifurcation position) had the poorest survival followed by the $2^{\text {nd }}, 3^{\text {rd }}$, and $4^{\text {th }}$ quartiles (highest bifurcation position), respectively.

Table 3 presents the Cox proportional hazard regression of AIBD (continuous and quartiles) for incident CVD events. In model 1 (unadjusted), the per-standard deviation increase in AIBD (higher bifurcation position) was significantly associated with reduced $\mathrm{ACM}(\mathrm{HR}=0.98,95 \% \mathrm{Cl}: 0.97-0.99$, $P<0.01)$ but not significantly associated with a decreased hazard for CHD (HR $=0.99,95 \% \mathrm{Cl}$ : 0.97-1.0, $P=0.2)$ and CVD (HR $=0.99,95 \% \mathrm{Cl}: 0.99-1.0$, $P=0.06)$. Also in the unadjusted models, we found that decreasing AIBD quartiles (lower bifurcation position) were associated with stepwise increasing hazard for CHD, CVD, and ACM. Compared to the $4^{\text {th }}$ quartile of AIBD (highest bifurcation position), individuals in the 1st quartile (lowest bifurcation position) were 2.2 times as likely to experience ACM, and this increase was the only significant finding $(P$ $<0.01)$. After adjustments for traditional CVD risk factors of age, gender, ethnicity, smoking, diabetes, SBP, hypertension medication use, and total and HDL cholesterol, however, the associations of AIBD (continuous and quartiles) for ACM were no longer significant.

\section{Discussion}

In a multi-ethnic cohort of community-living older adults, we found that a smaller AIBD (lower

Table 1. Cohort characteristics by AIBD quartile.

\begin{tabular}{|c|c|c|c|c|c|c|}
\hline $\mathrm{N}=1,711$ & Cohort & Q1 & Q2 & Q3 & Q4 & $P$ \\
\hline $\mathrm{AIBD}, \mathrm{mm}$ & $26(15)$ & $7(9)$ & $21(3)$ & $31(3)$ & $45(8)$ & $<0.01$ \\
\hline Age, y & 65 (10) & $69(9)$ & $65(10)$ & $63(9)$ & $61(9)$ & $<0.01$ \\
\hline Male & 867 (51\%) & $54 \%(50)$ & $49 \%(50)$ & $49 \%$ (50) & $51 \%(50)$ & 0.37 \\
\hline \multicolumn{7}{|l|}{ Ethnicity } \\
\hline Caucasian & 725 (42\%) & $43 \%(50)$ & $42 \%(49)$ & $40 \%$ (49) & $45 \%(50)$ & 0.48 \\
\hline Hispanic & $427(25 \%)$ & $24 \%(43)$ & $26 \%(44)$ & $27 \%(44)$ & $23 \%(42)$ & 0.57 \\
\hline African & $352(21 \%)$ & $22 \%(42)$ & $18 \%(38)$ & $21 \%(41)$ & $21 \%(41)$ & 0.45 \\
\hline Chinese & 207 (12\%) & $11 \%(31)$ & $14 \%(35)$ & $12 \%(33)$ & $11 \%(31)$ & 0.29 \\
\hline Smoking & 861 (50\%) & $58 \%(49)$ & $49 \%(50)$ & $45 \%(50)$ & $50 \%(50)$ & $<0.01$ \\
\hline Diabetes & $215(13 \%)$ & $12 \%(32)$ & $11 \%(31)$ & $14 \%(35)$ & $13 \%(34)$ & 0.63 \\
\hline Systolic blood pressure, mm Hg & $127(21)$ & $130(22)$ & $127(22)$ & $126(22)$ & $123(19)$ & $<0.01$ \\
\hline Hypertension medication & $35 \%$ & $39(49)$ & $35(48)$ & $34(47)$ & $32(47)$ & 0.22 \\
\hline Total cholesterol, mg/dL & $196(35)$ & $193(35)$ & $196(34)$ & $195(51)$ & $199(35)$ & 0.09 \\
\hline HDL cholesterol, mg/dL & $51(15)$ & $51(16)$ & $52(15)$ & $50(15)$ & $51(15)$ & 0.5 \\
\hline
\end{tabular}

$\mathrm{ACM}=$ all-cause mortality; $\mathrm{AIBD}=$ aorto-iliac bifurcation distance; $\mathrm{HDL}$ = high-density lipoprotein; $\mathrm{Q}=$ quartile.

Data are means (SD), n (\%). P represents quartile comparisons. 
bifurcation position) was significantly associated with increased hazard for incident ACM. We also found that this increased risk was explained by traditional CVD risk factors. No significant associations were observed between the AIBD and incident CHD and CVD; although, their trends were similar to that of ACM.

Prior studies have reported a lower aorto-iliac bifurcation position with older age (age-related bifurcation descent) $[4,5]$. We were the first to report that in

Table 2. Log-rank survival test among AIBD quartiles for CVD events.

\begin{tabular}{lcc}
\hline $\mathbf{N}=\mathbf{1 , 7 1 1}$ & $\chi^{\mathbf{2}}$ & $\boldsymbol{P}$ \\
\hline CHD, 63 (3.7\%) & 2.1 & 0.5 \\
CVD, 100 (5.8\%) & 3.8 & 0.3 \\
ACM, 129 (7.5\%) & 15 & $<0.01$ \\
\hline
\end{tabular}

$\mathrm{ACM}=$ all-cause mortality; $\mathrm{AIBD}=$ aorto-iliac bifurcation distance; $\mathrm{CHD}=$ coronary heart disease (defined as myocardial infarction, resuscitated cardiac arrest, and sudden cardiac death); CVD = cardiovascular disease (defined as CHD plus stroke and stroke death). two separate cohorts, one clinical and the other community-based, CVD risk factors were independently associated with age-related bifurcation descent [1, 2]. Furthermore, in a multi-ethnic cohort, we reported that atherosclerotic risk factors of age, gender, hypertension, and smoking were independently associated with a lower bifurcation position. In contrast, risk factors that are commonly associated with arterial stiffness, such as diabetes and elevated triglycerides, were associated with a higher bifurcation position. We surmised that a lower bifurcation position may be an independent marker for vascular aging and hypothesized that a lower bifurcation position may also be an independent marker for incident CVD events.

Supportive of our hypothesis, we found that decreasing AIBD quartiles (lower bifurcation position) were associated with stepwise increasing risk for incident CHD, CVD, and ACM. These findings, however, were only significant in unadjusted models for ACM only, as the associations were attenuated after adjustments for common CVD risk factors. With older age, atherosclerotic changes in the aorta are associated with increasing aortic diameter

Table 3. Unadjusted and adjusted Cox proportional hazard ratios of AIBD quartiles for CVD events.

\begin{tabular}{|c|c|c|c|c|c|c|}
\hline \multirow[b]{2}{*}{$N=1,711$} & \multicolumn{2}{|c|}{ CHD } & \multicolumn{2}{|c|}{ CVD } & \multicolumn{2}{|c|}{ ACM } \\
\hline & HR $(95 \% \mathrm{Cl})$ & $P$ & HR $(95 \% \mathrm{Cl})$ & $P$ & HR $(95 \% \mathrm{Cl})$ & $P$ \\
\hline \multicolumn{7}{|l|}{ Unadjusted model } \\
\hline AIBD, mm, per SD & $0.99(0.97-1.0)$ & 0.2 & $0.99(0.98-1.0)$ & 0.06 & $0.98(0.97-0.99)$ & $<0.01$ \\
\hline Q4 ( $\geq 36$ mm) & 1 [Ref] & & 1 [Ref] & & 1 [Ref] & \\
\hline Q3 (26-36 mm) & $1.0(0.5-2.1)$ & 1.0 & $1.1(0.6-2.0)$ & 0.8 & $1.0(0.6-1.8)$ & 1.0 \\
\hline Q2 (17-26 mm) & $1.1(0.5-2.4$ & 0.8 & $1.2(0.7-2.1)$ & 0.6 & $1.5(0.9-2.6)$ & 0.1 \\
\hline $\mathrm{Q} 1(\leq 17 \mathrm{~mm})$ & $1.5(0.8-3.0)$ & 0.2 & $1.6(0.9-2.7)$ & 0.1 & $2.2(1.3-3.6)$ & $<0.01$ \\
\hline \multicolumn{7}{|l|}{ Adjusted model* } \\
\hline AIBD, mm, per SD & $1.0(0.99-1.0)$ & 0.8 & $1.0(0.98-1.0)$ & 1.0 & $0.99(0.98-1.01)$ & 0.8 \\
\hline $\mathrm{Q} 4$ ( $\geq 36 \mathrm{~mm})$ & 1 [Ref] & & 1 [Ref] & & 1 [Ref] & \\
\hline Q3 (26-36 mm) & $0.8(0.4-1.7)$ & 0.6 & $0.9(0.5-1.6)$ & 0.7 & $0.9(0.5-1.6)$ & 0.7 \\
\hline Q2 (17-26 mm) & $0.9(0.4-1.8)$ & 0.7 & $1.0(0.5-1.8)$ & 0.9 & $1.1(0.7-2.0)$ & 0.6 \\
\hline $\mathrm{Q} 1(\leq 17 \mathrm{~mm})$ & $0.8(0.4-1.7)$ & 0.5 & $0.9(0.5-1.7)$ & 0.8 & $1.1(0.6-1.8)$ & 0.8 \\
\hline
\end{tabular}

$\mathrm{ACM}=$ all-cause mortality; $\mathrm{AIBD}=$ aorto-iliac bifurcation distance; $\mathrm{CHD}=$ coronary heart disease (defined as myocardial infarction, resuscitated cardiac arrest, and sudden cardiac death); $\mathrm{Cl}=$ confidence interval; $\mathrm{CVD}=$ cardiovascular disease (defined as CHD plus stroke and stroke death); $\mathrm{HR}=$ hazard ratio; $\mathrm{Q}=$ quartile.

*Model adjusted for age, gender, ethnicity, smoking, diabetes, systolic blood pressure, hypertension medications, and total and high-density lipoprotein cholesterol. 
$[6,7]$. Mechanisms that increase the aortic diameter which age are thought to also result in age-related bifurcation descent; however, older age is also associated with aortic stiffness, which may restrict these changes [8]. In the pathology of abdominal aortic aneurysms, which occur with an excessive abdominal aortic diameter increase that can result in rupture and death, atherosclerotic risk factors are associated with increased aortic diameter, but risk factors for aortic stiffness are associated with decreased aortic diameter $[9,10]$. We reported similar observations with the AIBD and surmised that the same process may underlie abdominal aortic elongation as suggested by age-related bifurcation descent. The opposing effects of atherosclerosis and stiffness in the aorta may, in part, explain our null findings. Most CVD risk factors were associated with a decreased ABID distance, but CVD risk factors that are also associated with stiffness were associated with an increased distance (less aortic descent), ultimately reducing the value of the ABID distance as an overall risk marker.

Strengths of our study include the use of a multi-ethnic cohort and adjudicated CVD events; however, our study also has important limitations. Prevalent CVD at baseline was an exclusion criterion, and thus, results may differ in persons with clinically apparent CVD. Also, we acknowledge the current limited clinical utility of our findings because ul- trasonography, the primary screening modality for aortic disease, is a proven and more cost-effective method without radiation exposure. Importantly, our results have strong research implications for anatomical changes in vascular beds, and if coupled with advancements in diagnostic imaging, these findings may aid future clinical use.

In conclusion, in a multi-ethnic cohort of community-living, healthy older adults, a lower bifurcation position was not independently associated with CVD events. The opposing effects of atherosclerosis and stiffness in the aorta may, in part, explain our null findings.

\section{Acknowledgements}

This study was supported by grant HL072403 (Dr. Criqui) and contract N01HC95159 to NO1HC95169. Dr. Forbang was supported by a supplement to N01HC95190.

\section{Conflict of Interest}

The authors have no conflict of interest relevant to this publication.

\section{Comment on this Article or Ask a Question}

\section{References}

1. Forbang NI, Nguyen $\mathrm{T}$, Ix JH, Criqui $\mathrm{MH}$, Allison MA. The Downward Shift of the Aortic Bifurcation, a Possible Marker for Vascular Aging. J Surg Radiol. 2011;2:372377. PMID: 26702364

2. Forbang $\mathrm{NI}$, Ix JH, Allison MA, Criqui MH. Associations of cardiovascular disease risk factors and calcified atherosclerosis with aortoiliac bifurcation position: the multiethnic study of atherosclerosis. Angiology. 2015;66:90-5. DOI: 10.1177/0003319713516669.

3. Bild DE, Bluemke DA, Burke GL, Detrano R, Diez Roux AV, Folsom AR, et al. Multi-ethnic study of atherosclerosis: objectives and design. Am J Epidemiol. 2002;156:871881. DOI: 10.1093/aje/kwf113
4. Voboril R. [The position of the aortic bifurcation in humans]. Sborník Věd Pr Lékařské Fak Karlovy Univerzity V Hradci Král Suppl. 1993;36:87-104. PMID: 8191258

5. Kornreich L, Hadar H, Sulkes J, Gornish M, Ackerman J, Gadoth N. Effect of normal ageing on the sites of aortic bifurcation and inferior vena cava confluence: a CT study. Surg Radiol Anat SRA. 1998;20:638. DOI: 10.1007/BF01628118

6. Allison MA, Kwan K, DiTomasso D, Wright CM, Criqui MH. The epidemiology of abdominal aortic diameter. J Vasc Surg. 2008;48:121-127. DOI: 10.1016/j. jvs.2008.02.031.

7. Laughlin GA, Allison MA, Jensky $N$, Aboyans V, Wong ND, Detrano R, et al. Abdominal Aortic Diameter and Vascular
Atherosclerosis: The Multi-Ethnic Study of Atherosclerosis. Eur J Vasc Endovasc Surg 2011;41:481-487. DOI: 10.1016/j. ejvs.2010.12.015.

8. Mitchell GF, Conlin PR, Dunlap ME, Lacourcière $Y$, Arnold JMO, Ogilvie $\mathrm{RI}$, et al. Aortic Diameter, Wall Stiffness, and Wave Reflection in Systolic Hypertension. Hypertension. 2008;51:105-111. DOI: 10.1161/ HYPERTENSIONAHA.107.099721.

9. Golledge J, Muller J, Daugherty A, Norman P. Abdominal aortic aneurysm: pathogenesis and implications for management. Arterioscler Thromb Vasc Biol. 2006;26:2605-2613. DOI: 10.1161/01. ATV.0000245819.32762.cb 
10. Iribarren $C$, Darbinian JA, Go AS, Fireman $\mathrm{BH}$, Lee CD, Grey DP. Traditional and novel risk factors for clinically diagnosed abdominal aortic aneurysm: the Kaiser multiphasic health checkup cohort study. Ann Epidemiol. 2007;17:669-678. DOI: 10.1016/j.annepidem.2007.02.004
Cite this article as: Forbang $\mathrm{NI}$, Allison MA, Criqui MH. Lower AortoIliac Bifurcation Position and Incident Cardiovascular Disease: A Multi-Ethnic Study of Atherosclerosis (MESA) AORTA (Stamford). 2016;4(5):156-161. DOI: $\quad$ http://dx.doi.org/10.12945/j. aorta.2016.16.020 